\title{
Werner Paravicini, Rimvydas Petrauskas, Grischa Vercamer (dir.), Tannenberg - Grunwald - Žalgiris 1410 : Krieg und Frieden im späten Mittelalter
}

Wiesbaden : Harrassowitz (Deutsches Historisches Institut Warschau. Quellen und Studien, 26), 2012, 356 p., $52 €$

\section{Mathieu Olivier}

\section{(2) OpenEdition}

\section{Journals}

Édition électronique

URL : http://journals.openedition.org/ifha/7553

DOI : 10.4000/ifha.7553

ISSN : 2198-8943

Éditeur

IFRA - Institut franco-allemand (sciences historiques et sociales)

Référence électronique

Mathieu Olivier, « Werner Paravicini, Rimvydas Petrauskas, Grischa Vercamer (dir.), Tannenberg Grunwald - Žalgiris 1410 : Krieg und Frieden im späten Mittelalter », Revue de I'IFHA [En ligne], Date de recension, mis en ligne le 13 décembre 2013, consulté le 22 septembre 2020. URL : http:// journals.openedition.org/ifha/7553; DOI : https://doi.org/10.4000/ifha.7553

Ce document a été généré automatiquement le 22 septembre 2020.

(C)IFHA 


\section{Werner Paravicini, Rimvydas Petrauskas, Grischa Vercamer (dir.), Tannenberg - Grunwald - Žalgiris 1410 : Krieg und Frieden im späten Mittelalter}

Wiesbaden : Harrassowitz (Deutsches Historisches Institut Warschau. Quellen und Studien, 26), 2012, 356 p., $52 €$

\section{Mathieu Olivier}

Comme l'on pouvait s'y attendre, le $600^{\text {ème }}$ anniversaire de la bataille de Tannenberg (15 juillet 1410), qui vit l'armée polono-lituanienne triompher de l'ordre Teutonique, s'est soldé par un regain d'intérêt savant pour un événement qu'un certain nombre de sources allemandes $\mathrm{du} \mathrm{XV}^{\mathrm{e}}$ siècle déjà appelaient tout simplement la "grande bataille ». À preuve le colloque international qui s'est tenu à Vilnius en octobre 2010, et dont le présent volume est l'aboutissement. Dans le prolongement des travaux menés depuis une vingtaine d'années au moins, vingt et un historiens - parmi lesquels on notera la présence de Ph. Contamine et J.-M. Moeglin - s'efforcent d'arracher un peu plus encore le champ de bataille de Tannenberg/Grunwald/Žalgiris au paradigme anachronique de la «bataille des peuples» qui en a si longtemps gauchi l'interprétation. Disons tout de suite que le résultat est pour le moins concluant. Les questions relatives au déroulement de la bataille elle-même ne sont pas éclipsées (S. Ekdahl sur les mouvements de troupe et la question, toujours débattue, de la localisation exacte de l'affrontement; K. Militzer sur l'épisode célèbre des deux épées, rapporté notamment par le chroniqueur Jan Długosz), et quelques études reviennent, assez classiquement, sur les prémices et l'arrière-plan politique de l'affrontement décisif (S. Rowell sur le grand-duché de Lituanie aux premiers temps de l'union avec la Pologne, A. Dubonis sur l'évolution de la zone frontière entre Lituanie et terres de l'Ordre depuis la fin du XIII siècle, telle que les sources écrites mais surtout l'archéologie, permettent de la faire revivre). Plus nombreuses sont néanmoins les contributions qui prennent Tannenberg comme point de départ ou point d'ancrage 
chronologique pour aborder des aspects jusque-là délaissés par la médiévistique, comme les fortunes diverses du renseignement teutonique en terrain ennemi (S. Jozwiak), les instruments de la diplomatie polonaise (A. Sweda), la réalité de la contribution militaire de ceux que les sources de l'Ordre appellent tout simplement les «hommes libres» (G. Vercamer), ou bien encore le rythme de l'acculturation lituanienne en matière de technologie et de tactique militaires (D.Baronas). L'exploitation toujours plus intensive des riches archives de l'Ordre de Prusse permet par ailleurs de jeter une lumière nouvelle sur de vieilles questions, notamment celle du coût de la guerre de 1409 - 1410 (J. Sarnowski), ou bien celle du rôle des mercenaires des pays de la Couronne de Bohême dans ces mêmes années (U. Tresp). Le principal mérite de ce volume d'actes réside sans nul doute dans le fait qu'il ouvre à la bataille de Tannenberg de nouveaux horizons, à plus d'un titre. C'est en premier lieu l'horizon diachronique de la longue durée. Tandis que H. Sahanovic et A. Nikzentaitis s'intéressent après bien d'autres à la mémoire de Tannenberg dans l'Europe du CentreEst, sur un mode essayistique, Th. Wünsch fait une place de choix au principal protagoniste de la guerre des arguments entre l'Ordre et la Pologne, Paul Wladimiri, dans une généalogie de ce qu'il appelle l'école de pensée " réaliste » dans les relations internationales. Nombreuses sont par ailleurs les contributions qui proposent une remise en perspective bienvenue, à l'heure où la chose militaire est revenue au premier plan des recherches sur la fin du Moyen Âge. C'est tout l'intérêt des études complémentaires de Ph. Contamine, H.-H. Kortüm et M. Prietzel sur l'art de la guerre et les batailles au bas Moyen Âge. La contribution de J.-M. Moeglin, sur les sentences arbitrales rendues dans le litige entre la Pologne et l'Ordre, avant et encore après Tannenberg, procède d'une même ambition et confronte l'épais dossier prussien à d'autres procédures arbitrales mises en place plus à l'ouest. Par ce dernier objet d'études, nous touchons du doigt le dernier thème central du présent recueil, celui de la paix. C'est tout d'abord la " paix des braves " qu'il faut bien conclure en bonne et due forme une fois que les armes se sont tues (Kl. Neitmann sur l'art de conclure la paix en Prusse dans les années 1398-1435). C'est encore l'option diplomatique qui cherche à éviter la guerre jusqu'au dernier moment, ou à lui substituer la procédure amiable (A. Szweda et J.-M. Moeglin, déjà cités). Ce sont enfin - thème longtemps ignoré - les relations d'égal à égal, faites d'amitiés réelles et d'intérêts parfois communs, qui se tissent au fil des décennies entre les frères de l'Ordre et ces " païens » de Lituanie qui justifient leur engagement sur les bords de la Baltique : servies par une documentation abondante, les deux longues contributions de W. Paravicini et de R. Petrauskas, que l'on lira comme un diptyque, se penchent de près sur la trame de ces relations spéciales au tournant du XIV et du XV siècle, entre affinités électives et liaisons dangereuses. Dans le droit fil des réflexions liminaires de M. Kintzinger gageons que ce gros volume contribuera grandement à remettre en question la pertinence de la coupure entre l'Ouest et l'Est de l'Europe pour les derniers siècles du Moyen Âge. 
INDEX

Index chronologique : Mittelalter

Thèmes : Militärgeschichte

\section{AUTEUR}

MATHIEU OLIVIER

Lycée Dumont-d'Urville, Toulon 